Originalveröffentlichung in: Jahraus, Oliver ; Raß, Michaela Nicole ; Eberle, Simon (Hrsgg.): Sache/Ding : eine ästhetische Leitdifferenz in der Medienkultur der Weimrarer Republik, München 2017, S. 257-277

Henry Keazor

\title{
Versachlichung und Stasis als Zeichen der Kontrolle in Fritz Langs M (1931)
}

\section{M-Eine »Filmreportage«?}

Fritz Langs M (193I), so kann man in Beiträgen und Büchern zu dem Film immer wieder lesen, sei eine "Film-Reportage«. ${ }^{1}$ Diese Charakterisierung wie auch der Begriff selbst gehen - wie so oft - auf Lang selbst sowie seine Frau Thea von Harbou zurück: Lang erklärte nach der Fertigstellung von Die Frau im Mond, dass er sich nach diesem Science-Fiction-Abenteuer verstärkt der Realität zuwenden wolle, was zur Film-Reportage hinführe. Nicht, dass er ganz auf den fiktionalen Film verzichten wolle - aber er strebe danach, verstärkt aus dem Filmstudio herauszugehen und etwas ohne jeden Zierrat und ohne alle Übertreibung zu zeigen, so Lang in einem Interview mit dem Berliner Börsen-Courier vom I2. Oktober 1929. ${ }^{2}$ Dieses Bekenntnis zu einem schmucklosen Realismus lässt sich freilich nicht zu I0о \% auf $\mathrm{M}$ übertragen, denn im Unterschied zu Langs Idee von einem außerhalb des Studios gedrehten Film wurde M komplett im Studio gedreht; nichtsdestotrotz reklamierte Thea von Harbou dann für den Film, dass es sich dabei um einen "Bericht" handele, ${ }^{3}$ obwohl auch die verschiedenen, von Lang in $\mathrm{M}$ an den Tag gelegten narrativen, filmsprachlichen Raffinessen und Montagekunststücke eigentlich den Prinzipien eines den reinen Fakten verpflichteten, sachlichen Realismus widersprechen. ${ }^{4}$ Dessen ungeachtet beginnt selbst Siegfried Kracauer seinen ersten, am I8. Mai I93I unter dem Titel Unterwelt in der Frankfurter Zeitung erschienenen Artikel über M mit dem Hinweis, man liebe heute "Tatsachenberichte,${ }^{5}$ auch wenn er Lang dann nachweist, dass dieser - insbesondere gegen Ende des Films, wenn der Mörder, von den Kriminellen gefasst und vor ein Tribunal gestellt, zu einem schauspielerisch von Peter Lorre bravourös umgesetzten Geständnis anhebt - letzten Endes vom Tonfall der Nibelungen nicht loskomme: Anstatt sich in der sozialen Wirklichkeit zu verankern, heroisiere und mythologisiere Lang die von ihm gezeigte Unterwelt, womit er "den Nutzwert der vorangegangenen Reportagen « wieder tilge. ${ }^{6}$ Insbesondere aber der Ansatz von Lotte Eisner, die M I976 mit der Strömung der Neuen Sachlichkeit in Beziehung brachte, hat lange nachwirkende Folgen hinsichtlich der Rezeption des Films gezeitigt. ${ }^{7}$ 
Dies ist sicherlich auch auf Langs geschickte Strategie zurückzuführen, dass M zeitgleich mit dem spektakulären Mordprozess um den Serienmörder Peter Kürten, den »Vampir von Düsseldorf", in die Kinos $\mathrm{kam}^{8}$ und dass es immer wieder sehr didaktisch angelegte Sequenzen in Langs Film gibt, die ein wenig in ihrer Erklärung polizeilicher Ermittlungsarbeit an zeitgenössische Dokumentarfilme erinnern. Freilich besteht hier eigentlich ein grundlegender Unterschied zwischen Dokumentar- und Reportagefilm, denn während Letzterer mit vorwiegend spontan gedrehtem und dann per Montage entsprechend aufbereitetem Material arbeitet, hatte sich für den Dokumentarfilm schon in den I920er Jahren die Praxis etabliert, dass zu dessen Vorbereitung regelrechte Drehbücher verfasst wurden, ${ }^{9}$ womit die Grenzen zwischen fiktionalem und dokumentarischem Film bereits aufgeweicht waren. Weitere Faktoren, die eine solche strenge Unterscheidung zwischen fiktionalem und dokumentarischem Film z. T. schwer bis unmöglich machen, lassen sich hier anführen: angefangen von Entscheidungen, wie, wo und mit welchem Blickpunkt die Kamera aufgestellt wird, über die anschließende Montage des gedrehten Materials bis hin zu Überlegungen, dass ein dokumentarischer Film damit ebenso fiktionale Tendenzen aufweist wie umgekehrt ein fiktionaler Film auch als Dokument einer bestimmten Gesellschaft gesehen werden kann. Der US-amerikanische Filmtheoretiker Bill Nichols betrachtet fiktionale Filme z. B. unter dem Aspekt, dass sie gewissermaßen eine Dokumentation bestimmter, mit solchen Filmen erfüllter Wünsche und Sehnsüchte des Drehbuchautors und/oder des Regisseur und/oder der Produktionsfirma und/oder, so könnte man ergänzen, des Publikums sind, auf welche die Protagonisten mit ihren Filmen reagieren. ${ }^{10}$ Angesichts all dieser Berührungspunkte wurde der Begriff des Dokumentarfilms auch immer wieder kritisch diskutiert, auch wenn man es hier ganz pragmatisch mit dem schottischen Dokumentarfilmer John Grierson halten kann, der den Begriff documentary mit seiner Rezension von Robert Flahertys MOANA (I926) einführte, ${ }^{11}$ auch als "Vater des britischen und kanadischen Dokumentarfilms" verehrt wird und schon 1932 schrieb: "Documentary is a clumsy description, but let it stand «. ${ }^{12}$ In jedem Fall kann festgehalten werden, dass Langs $M$ in seiner Ästhetik sequenzweise eher etwas von einem didaktisch ausgerichteten Dokumentarfilm denn von einem echten Reportagefilm hat. Die seinerzeit aktuelle Thematik des Serienmörders Kürten in Kombination mit den Polizeiarbeit didaktisch vermittelnden Sequenzen ${ }^{13}$ sorgte jedoch offenbar dafür, dass diese Differenzierung keine Berücksichtigung fand und $\mathrm{M}$ daher, den Aussagen Langs und von Harbous folgend, teilweise als Reportagefilm aufgefasst wurde. 
Es soll hier auch nicht so sehr darum gehen, diese Charakterisierungen von $M$ eingehend zu erörtern und kritisch zu diskutieren, sondern vielmehr auf einen anderen Aspekt der Versachlichung in M hinzuweisen.

\section{II. „Objects as Subjects«}

Wie allseits bekannt, sind in Filmen - und zumal in fiktionalen Filmen - zu sehende Gegenstände nicht dem Zufall überlassen, sondern sie werden mit mehr oder weniger Detailliebe ausgesucht, zusammengestellt und gezeigt, und ihnen können daher in Filmen auf unterschiedlichen Ebenen und in verschiedener Intensität bestimmte Funktionen und Bedeutungen in Filmen zukommen: Hitchcock's Objects as Subjects. The Significance of Things on the Screen hat der Emeritus of Theatre and Dance der Southeast Missouri State University Marc Raymond Strauss seine 2016 erschienene Studie betitelt, in welcher er darlegt, wie Dinge, Objekte und Gegenstände in Alfred Hitchcocks Filmen dadurch "subjektiviert" werden, dass ihnen entscheidende Funktionen bei der Bedeutungsstiftung und der Charakterisierung von Personen wie Situationen zugewiesen werden. ${ }^{14}$ Bereits in Hitchcocks frühem Film The Lodger: A Story of the London Fog (1927) lässt sich dies beobachten, etwa, wenn man eine den ganzen Film durchziehende Motivik in den Fokus rückt, welche die paranoide Stimmung visualisiert, die in der Londoner Gesellschaft herrscht, nachdem diese durch eine Reihe von spektakulären Morden an jungen Mädchen aufgestört worden ist. Um das fast panische Bedürfnis nach permanenter Kontrolle und Überwachung zu visualisieren, das sich unter diesen Bedingungen (ähnlich wie vier Jahre später in Fritz Langs M) unter den Stadtbewohnern Bahn bricht, arrangiert Hitchcock immer wieder an und für sich unbelebte Objekte wie z. B. Bilderrahmen oder auch die Rückfenster eines Autos (also stets Dinge, die mit dem »Sehen"zu tun haben) in einer Weise, dass sie an Augen gemahnen (Abb. Ia + b). ${ }^{15}$

Geradezu einen eigenen Handlungsimpetus bekommen Objekte schließlich in Hitchcocks neun Jahre später entstandenem Film SABOTAGE (I936), der bei Strauss auch in Form eines Filmstills das Cover seines Buches ziert: Als die junge Winnie Verloc erfährt, dass es sich bei ihrem Ehemann Carl um einen für eine geheime Organisation tätigen Saboteur handelt, der zudem ihren kleinen Bruder Stevie auf dem Gewissen hat, den Verloc zum Boten einer in einem Paket versteckten Bombe gemacht hatte, die - da Stevie sich verspätete - in seinen Händen explodierte, gewinnt das Besteck, mit dem sie ihrem Mann das Abendessen zurechtschneiden will, förmlich ein Eigenleben, 


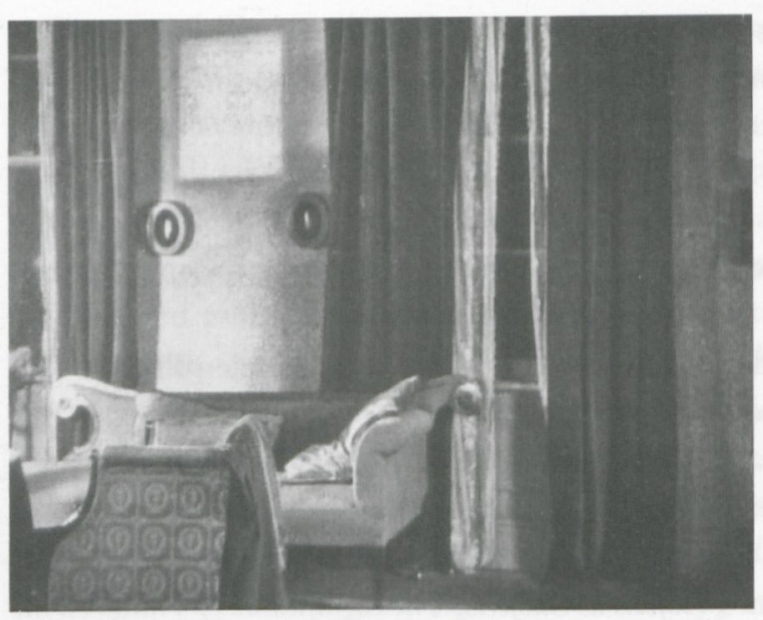

Abb. $1 \mathrm{a}+\mathrm{b}$ :

Alfred Hitchcock:

THE LOdger. A Story of

THE LONDON FOG (1927)

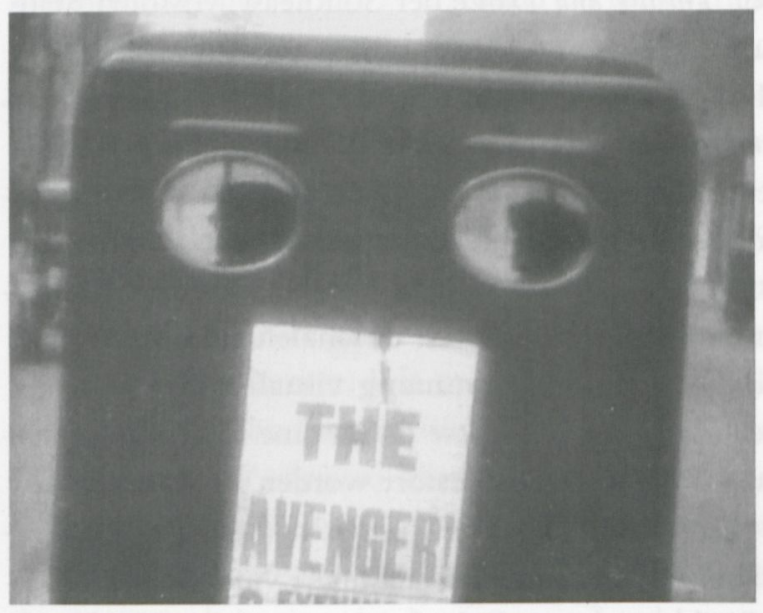

und es wirkt daher geradezu so, als töte nicht sie, sondern das Tranchiermesser von selbst ihren verräterischen Ehemann (dies wird von der auf dem Cover abgebildeten Szene, in der Winnie am Esstisch von zwei Kerzen, wie auf einem Altar, eingerahmt wird, vorab schon angedeutet). ${ }^{16}$

\section{III. "Subjects as Objects«: Die Macht der Kartografie}

Man könnte nun den Titel von Strauss' Buch entsprechend auf Langs $M$ hin adaptieren und das heißt hier: umkehren. Denn in M geschieht teilweise das genaue Gegenteil zu dem von Strauss bei Hitchcock beschriebenen Prozess: 
Lang's Subjects as Objects. The Significance of Things on the Screen in >M<, könnte man die Überschrift entsprechend anpassen, denn in Langs Film lässt sich immer wieder auch eine Versachlichung von natürlichen bzw. menschlichen Kontexten beobachten, die zum Zwecke einer Objektivierung (im Sinne von Objektwerdung) zugleich stillgestellt, d. h. in Stasis versetzt werden.

Ich möchte dies im Folgenden anhand des Motivs der Karte, des Plans und des Diagramms darlegen, von dem aus sich die verschiedenen Arten der Speicherung, Kommunikation und Verfügbarmachung von Wissen über Menschen und Dinge durch den Film hindurchverfolgen lassen.

In seinen Büchern The Power of Maps (1992) und Rethinking the Power of Maps (20I0) ${ }^{17}$ hat der amerikanische Kartograf und Kartografiehistoriker Denis Wood dargelegt, dass und inwiefern Karten nicht einfach nur Darstellungen der realen Welt sind. Sie erweisen sich bei eingehenderer Betrachtung vielmehr als sozial konstruierte Artefakte, die auf konsistenten semiotischen Codes basieren - und sie haben sehr viel (wie es die Titel von Woods Büchern bereits nahelegen) mit Macht und Kontrolle zu tun.

In der Tat differenzierte Woods britischer Kollege John Brian Harley bereits 1989 zwischen einer externen und einer internen Macht der Kartografie, wobei er die externe Macht in zweifacher Weise definierte: ${ }^{18}$ Auf der einen Seite, so Harley, können Karten als ein Spiegelbild der Macht gesehen werden, die auf die Kartografie ausgeübt wird. Somit spiegeln Karten vor allem die Interessen derjenigen wider, welche die Produktion solcher Karten in Auftrag geben. Auf der anderen Seite können Karten auch als ein Spiegelbild der Macht angesehen werden, die mithilfe der Kartografie ausgeübt wird: Karten werden z.B. in den unterschiedlichsten Kontexten verwendet, um Machtansprüche zu legitimieren - sie fungieren hierbei als die grafische Manifestation der Gültigkeit von Gesetzen, die eine herrschende Rechtsmacht erlassen hat bzw. sie bekräftigen und visualisieren generell bestehende Machtverhältnisse.

In diesem Zusammenhang wird sodann auch die interne Macht deutlich, die Karten innewohnen kann. So verdeutlicht Harley, auf welche Weise diese interne Macht Kartografen die Möglichkeit gibt, Macht und Kontrolle auszuüben: "Maps are a technology of power, and the key to internal power is cartographic process" (so Harley in einem späteren, ebenfalls »Deconstructing the Map « betitelten Aufsatz von 1992). ${ }^{19}$ Er denkt hierbei offenbar daran, dass die Fähigkeit, Karten zu erstellen, zugleich die Verfügung darüber impliziert, was in Karten aufgenommen oder ausgeschlossen wird, was wiederum dem Autor bzw. der Autorin einer Karte die Möglichkeit gibt, die mit einer Karte vermittelten Botschaften gemäß ihrer jeweiligen Interessen bzw. der Inter- 
essen ihrer Auftraggeber zu manipulieren (womit dann auch der Bereich der externen Macht erreicht wäre).

\section{Karten, Pläne und Diagramme als Mittel der Macht und Kontrolle}

Als solche Mittel der Machtausübung und Kontrolle werden Karten, Pläne und Diagramme in Langs $\mathrm{M}$ auch gezeigt: Sie fixieren die dynamische Wirklichkeit, halten sie in den erwähnten konsistenten semiotischen Codes fest und reduzieren sie zugleich auf das, was als wesentlich angesehen wird. Indem eine komplexe, lebendige Realität so erstarrt und vereinfacht lesbar gemacht
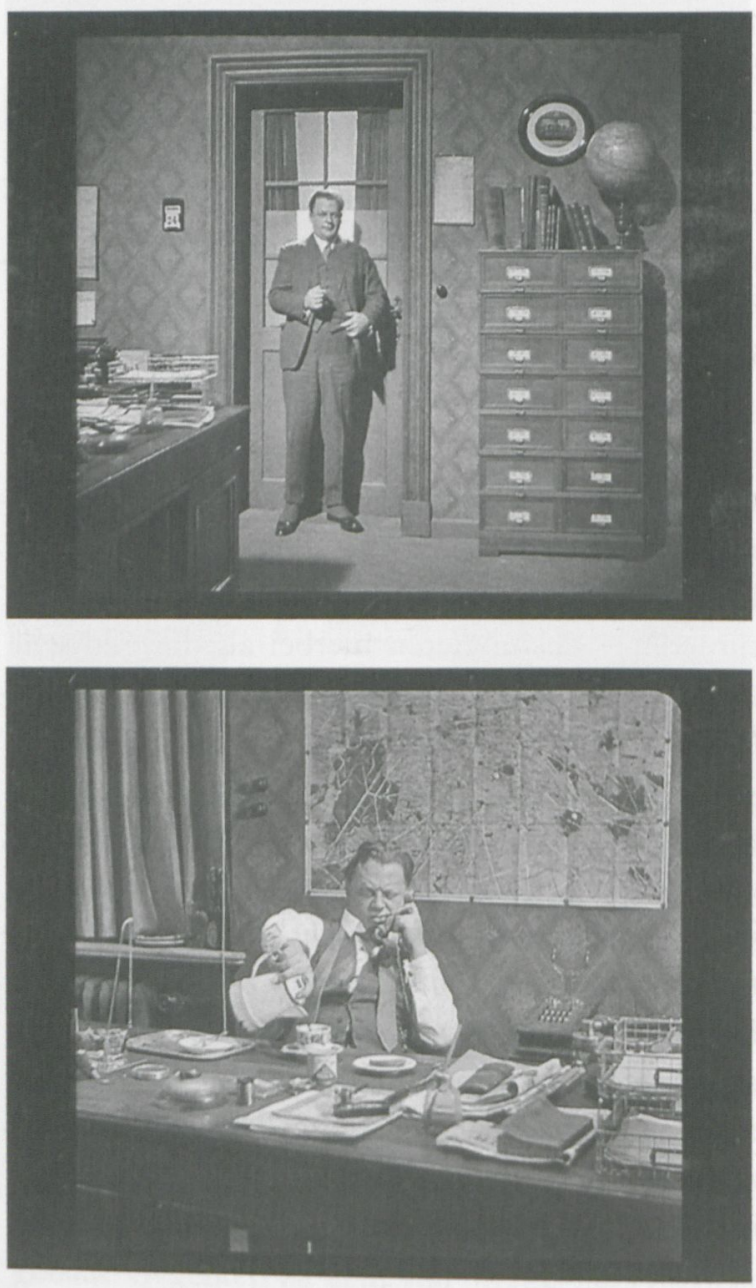

Abb. $2 \mathrm{a}+\mathrm{b}$ :

Fritz Lang: M (1931) 
wird, soll sie zugleich überschaubar und damit handhab- bzw. beherrschbar gemacht werden. Als Zeichen dieser Beherrschbarkeit und Beherrschung trifft man in Langs Film immer wieder Globen und Welt-, Land- und Stadtkarten in den Büros der Polizei an (Abb. 2a +b).

Zudem werden eben solche kartografischen und diagrammatischen Darstellungen der Wirklichkeit auch just ins Feld geführt, wenn es darum geht, dem scheinbaren Versagen der Polizei angesichts des sich ihrem Zugriff entziehenden Massenmörders argumentativ etwas entgegenzusetzen. Die gezeigten Karten, die sich über die fragmentarisch eingeblendete Realität legen und in die mithilfe eines riesigen Zirkels konzentrische Kreise geschlagen werden, sollen - jenseits des stetig erweiterten Suchradius um einen spezifischen Ort - suggerieren, dass der Täter so im wahrsten Sinne des Wortes

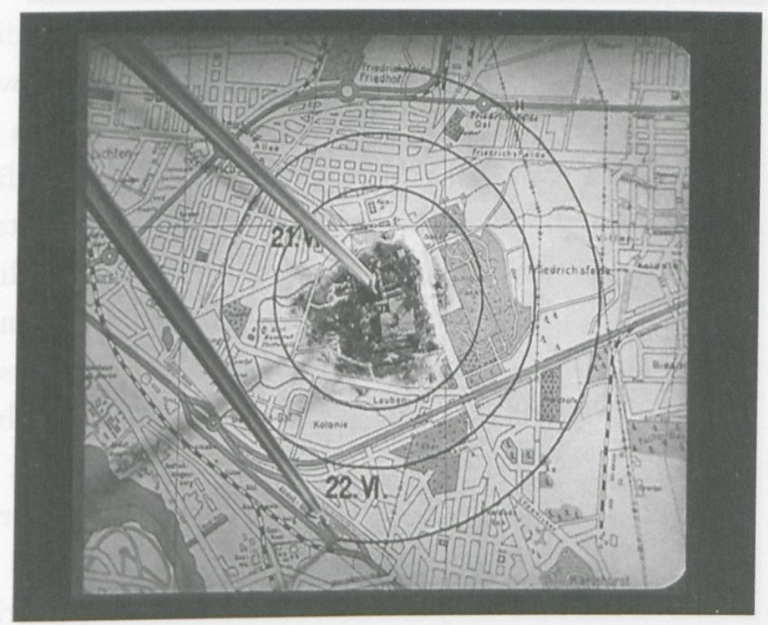

Abb. $3 a+b$ :

Fritz Lang: M (1931)

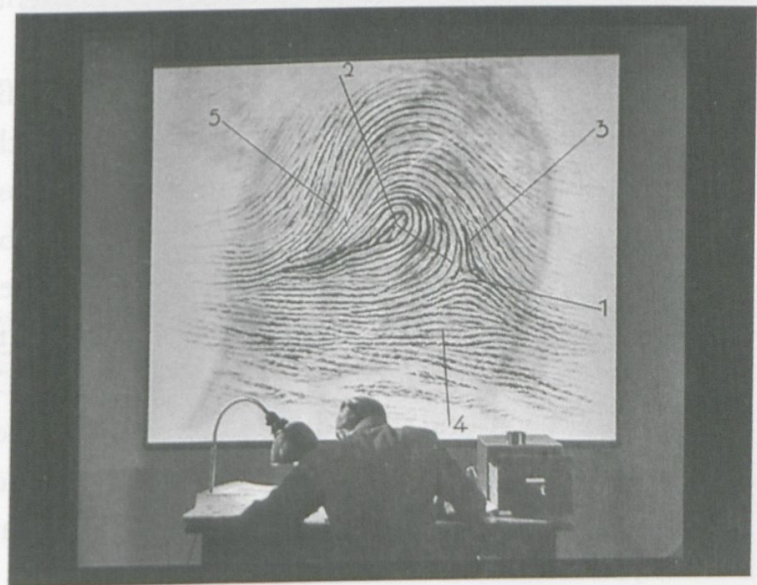


eingekreist wird, und fungieren so zugleich als Beschwörung der nach wie vor von der Polizei ausgeübten Kontrolle (Abb. 3a): Nicht umsonst illustriert die Sequenz eine Verteidigung des Polizeipräsidenten gegenüber einem Minister, der sich darüber beschwert, dass die Polizei den Mörder noch nicht dingfest gemacht hat. ${ }^{20}$ Dazu passt, dass dann selbst die im Zuge der Ermittlungen fixierten Fingerabdrücke (auch dies eines der "didaktischen" Elemente in Langs Film) ${ }^{21}$ etwas von einer kartografischen Darstellung erhalten (Abb. 3b).

Darüber hinaus erweisen sich die Karten und Pläne als etwas, das die beiden im Film den Mörder suchenden Parteien - auf der einen Seite die Polizei, auf der anderen Seite das organisierte Verbrechen - miteinander verbindet und zwar in expliziter wie impliziter Weise. Denn Lang assoziiert beide Seiten sowohl auf der Ebene des Dialogs als auch der Bilder, wie etwa an der berühmten Szene deutlich wird, in welcher der Anführer der Verbrecher, Schränker, einen Satz und eine Geste beginnt, die sodann mittels eines Match-Cuts von dem Polizeipräsidenten vollendet wird; ${ }^{22}$ zum anderen wenden beide Parteien sehr ähnliche Verfahrensweisen an, die jeweils auf systematische Beobachtung und Durchsuchung von verdächtigen Individuen und Personen setzen und hierfür jeweils einen großen Stab an Mitarbeitern mobilisieren: Auf der Seite der Ordnungsmacht die Polizisten, auf der Seite des Verbrechens die Bettler, die geradezu generalstabsmäßig erfasst und mit spezifischen Observierungsaufgaben in klar zugewiesenen Distrikten betraut werden. Implizit wird die Parallele zudem dadurch noch betont, dass auch aufseiten des organisierten Verbrechens Pläne, z. B. von Verkehrsverbindungen, im Hintergrund hängen, und wenn Schränker dazu aufruft, dem Mörder das Handwerk zu legen, da er die Aktivitäten der normalen Verbrecher durch die von ihm provozierte, erhöhte Polizeipräsenz behindere, legt er seine behandschuhte Hand in einer besitzergreifenden Geste just auf einen Plan der Stadt (Abb. $4 a-c) .^{23}$

Auch hinsichtlich sich daran sozusagen anschließender, weiterer Formen von Verfügung und Kontrolle ähneln sich die Methoden der beiden Seiten, denn sie arbeiten z. B. beide mit Adressbüchern, Listen, Verzeichnissen und Akten, in denen ebenfalls eine "Versachlichung" beobachtet werden kann, indem die Existenzen von Individuen hier auf bestimmte Parameter und Daten reduziert und diese fixiert, kommuniziert und zum Zweck von Kontrollen verfügbar gemacht werden (Abb. $5 \mathrm{a}-\mathrm{d}$ ): Die Adressbücher dienen dem Zweck, nach und nach die Bewohner aller Viertel aufzusuchen und zu verhören; zudem verfügen Polizisten wie Kriminelle über die Unterlagen und Schaltpläne zu einem bestimmten Gebäude und dessen Sicherheitssystemen (Abb. 6a-c). 

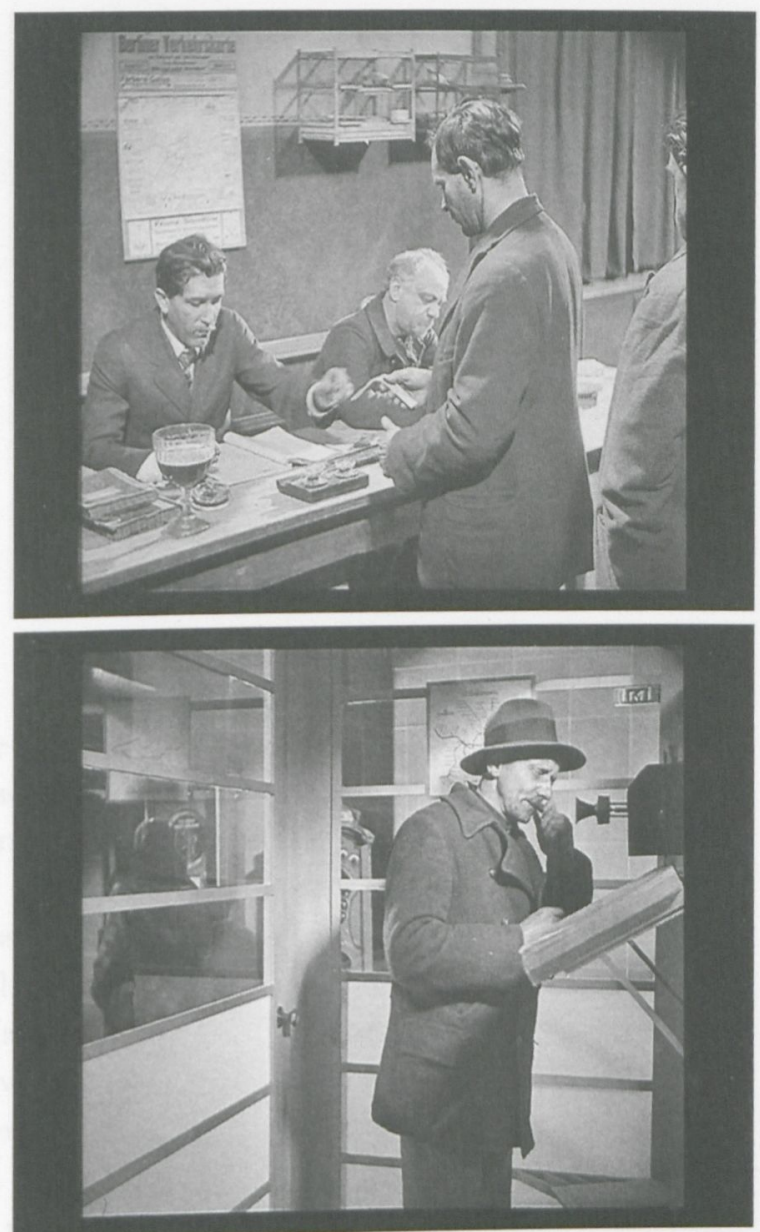

Abb. $4 a-c:$

Fritz Lang: M (1931)

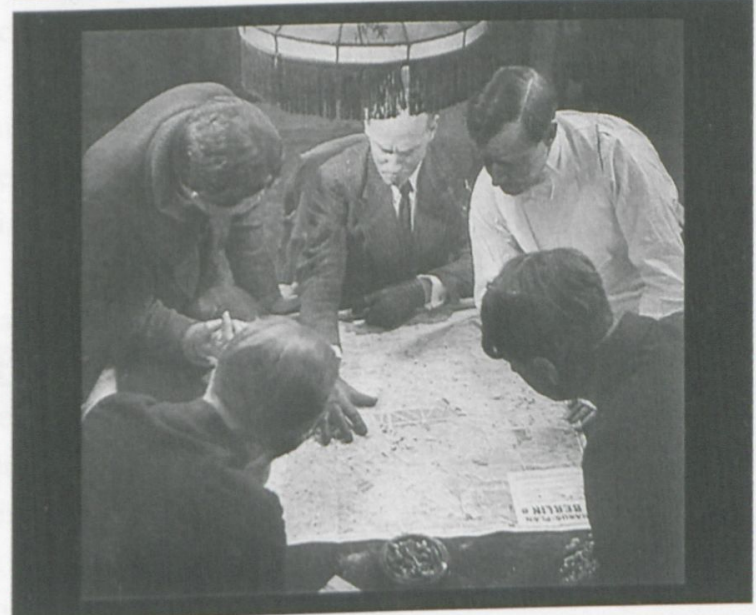




\section{Henry Keazor}
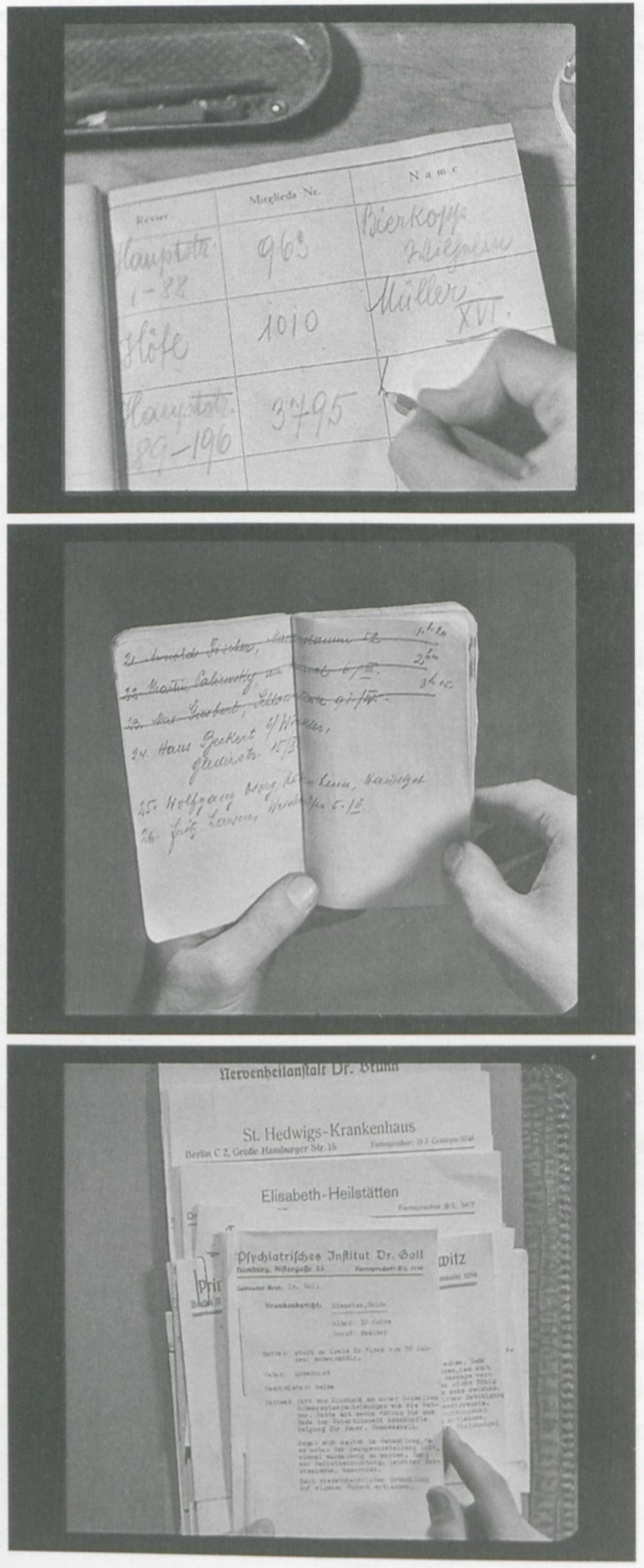

Abb. $5 a-c$ :

Fritz Lang: M (1931) 
Abb. 5d:

Fritz Lang: M (1931)

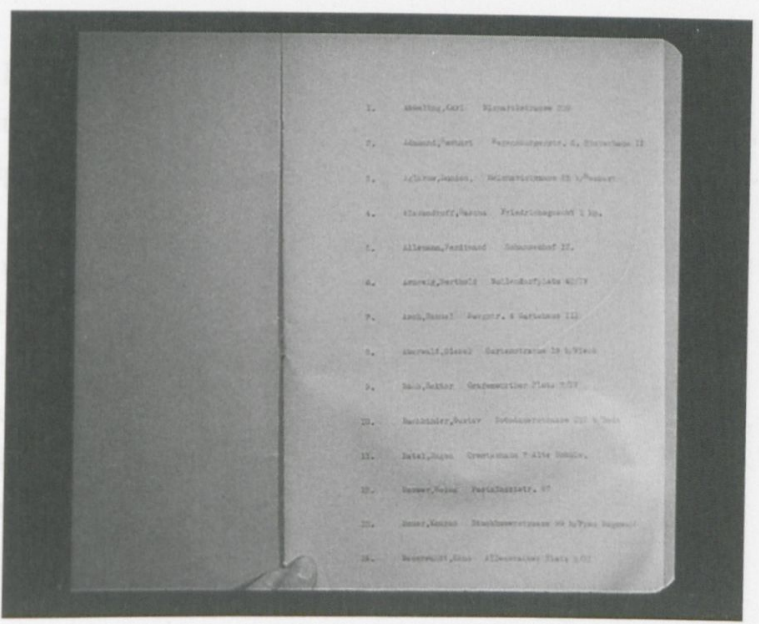

Allerdings lassen sich die beiden Seiten von Polizei und organisiertem Verbrechen noch in einer anderen Hinsicht bezüglich ihres Rekurses auf derartige Versachlichungen und Mittel der Kontrolle in Parallele zueinander setzen: Denn auch wenn sie ein Stück weit damit erfolgreich sind (der Mitarbeiter des Kommissars Lohmann kann schließlich die Wohnung des Mörders und damit auch seinen Namen - Hans Beckert - identifizieren, sodass sie das Haus beschatten können; die Kriminellen können Beckert, der indes in der Stadt unterwegs ist, dank ihres gut organisierten Systems aus Spitzeln auf seiner sodann erfolgenden Flucht bis in ein Bürohaus hinein verfolgen und dort dann dingfest machen), so geht die tatsächliche Identifikation von Beckert (d.h. nicht nur als Name und als Bewohner eines Hauses, sondern als reale physische Person) letzten Endes ausgerechnet auf einen Blinden zurück, für den all die zuvor eingesetzten Mittel der Informationsvermittlung - lesbare Karten, Pläne, Listen etc. - unzugänglich sind: Da er nur auf sein Gehör sowie seinen Tastsinn vertrauen kann, ist er es, der den Mörder an der von ihm immer wieder in Momenten der Erregung obsessiv gepfiffenen Melodie von Griegs In der Halle des Bergkönigs erkennt und damit seine Verfolgung veranlassen kann. Dass es Lang ein Anliegen ist, dieses besondere akustische Vermögen des Blinden herauszustellen ( $M$ war Langs erster Tonfilm und der den Mörder über das gepfiffene Lied identifizierende Blinde ist natürlich ein probates Mittel, die Leistungen des Tonfilms gegenüber dem Stummfilm herauszustellen, in dem diese Art der Identifizierung schwerfällig bis gar nicht vermittelbar gewesen wäre), ${ }^{24}$ wird deutlich, wenn er einen der von den Kriminellen zur Observierung auf die Straßen geschick- 

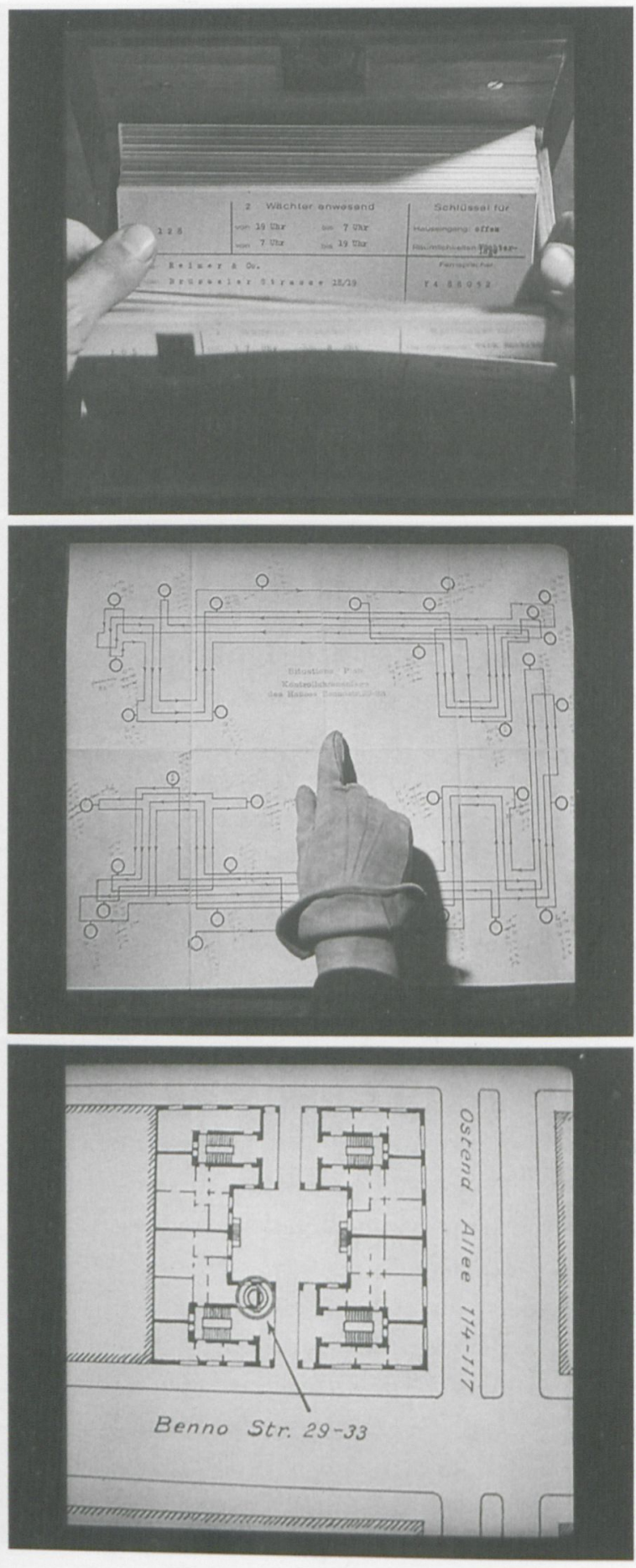

Abb. 6a-c:

Fritz Lang: M (1931) 
ten Bettler als "falschen« Blinden agieren lässt, der natürlich als im direkten Kontrast zu dem echten Blinden verstanden werden soll. ${ }^{25}$

Darüber hinaus deutet Lang in einzelnen Momenten des Films auch an, wieso die zunächst verfolgte Strategie der Kontrollgewinnung über den Mörder auch ein Stück weit scheitert: Mit der Versachlichung der Phänomene geht nicht nur eine Reduktion, sondern auch eine gewisse Erstarrung und Stasis, eine Immobilisierung einher, welche die so erlangten Resultate der Komplexität und vor allem: der Dynamik der realen Phänomene unterlegen macht. Denn interessanterweise zeigt uns Lang selbst immer wieder Dinge, die durchaus nicht statisch sind - man denke z. B. an die dynamischen Schaufensterauslagen, in denen entweder sich drehende Scheiben oder auf und ab schwingende Pfeile auf die Waren hinweisen oder aber diese sich selbst in frenetischer Bewegung befinden (Abb. $7 \mathrm{a}+\mathrm{b}$ ). Nicht zufällig sind diese

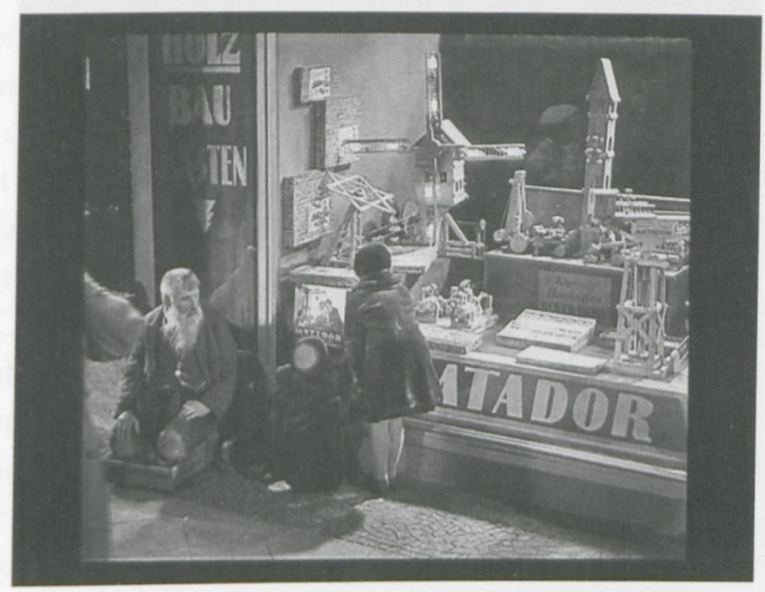

Abb. $7 a+b$ :

Fritz Lang: M (1931)

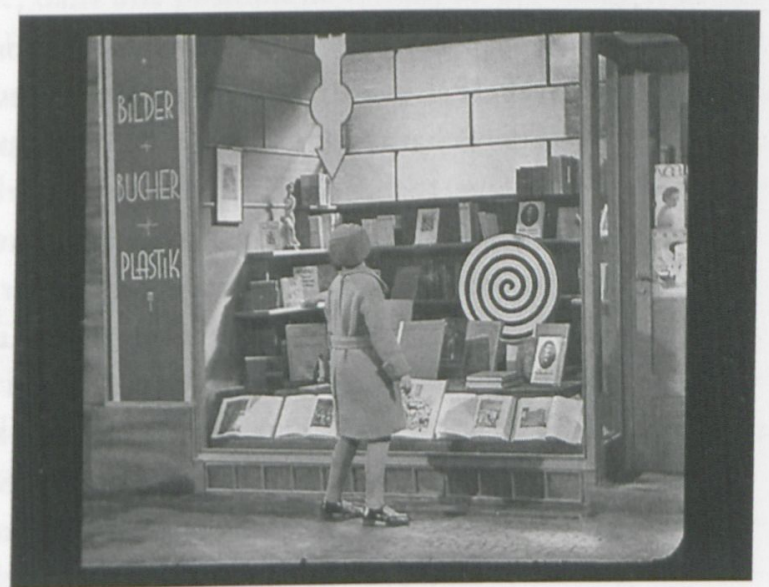




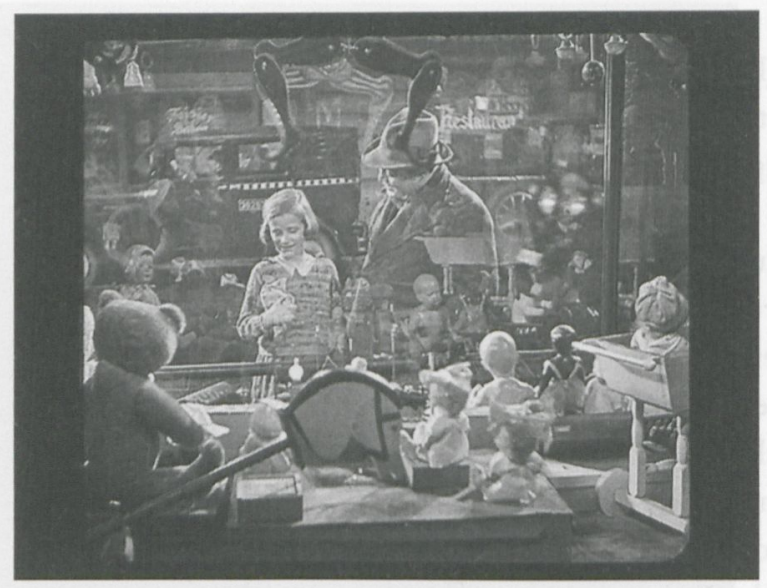

Abb. $7 c+d:$

Fritz Lang: M (1931)

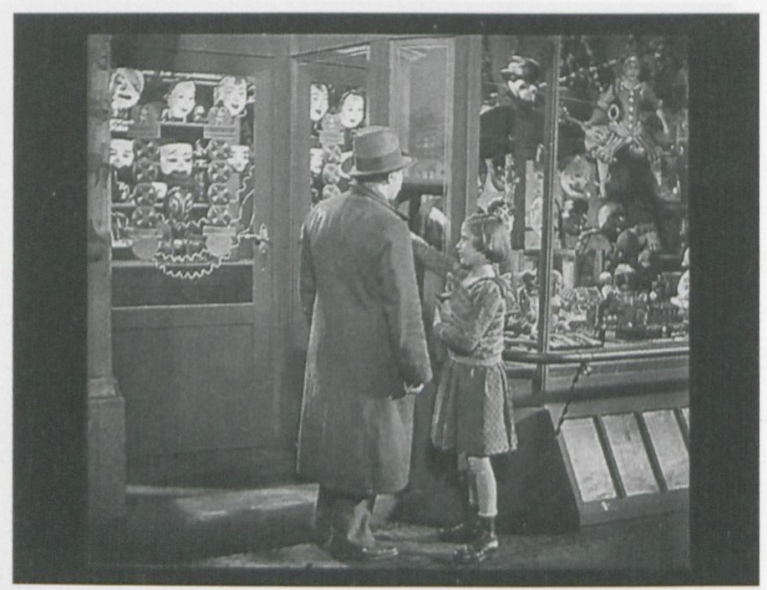

Lebendigkeit bzw. Erregung visualisierenden Dekorationen in Langs Film ${ }^{26}$ mit den von Beckert als seine potenziellen Opfer ausersehenen Kinder assoziiert, auf welche die Schaufensterdekorationen wie Lockvögel wirken (in einer Szene des Films lauert er einem Mädchen an einer solchen Auslage auf, ein anderes Mal führt er ein Mädchen zu einer solchen Auslage hin: Abb. $7 c+d) .{ }^{27}$

Lang stellt so dieser mit Verlockung und Gefahr assoziierten Dynamisierung von eigentlich toten Objekten die Erstarrung und Fixierung von lebendigen, natürlichen und menschlichen Phänomenen der Wirklichkeit in Karten, Plänen und Listen gegenüber, die dann nicht zufällig ebenfalls partiell dynamisiert werden müssen, um mit der Realität Schritt halten zu können: Nachdem man eine Tüte mit Resten von Süßigkeiten in der Nähe eines Tatorts gefunden hat, durchsucht man systematisch die Umgebung - und 
muss dazu immer weitere Kreise ziehen, da die Suche zunächst nichts ergibt. ${ }^{28}$ Und der bewegliche und sich durch die Stadt bewegende Mörder wird zuletzt eben nicht mithilfe dieser statischen Mittel gefasst, sondern dank des Gehörs und der Kombinationsgabe eines Blinden.

\section{V. "Parcours« vs. »carte«}

Dieser Unterschied zwischen einer statischen, Überblick und damit Kontrolle suchenden Position und einer beweglichen, sich die eigene Route erst im Vollzug suchenden Haltung lässt sich mit zwei Konzepten des Umgangs mit Raum fassen, die der französische Philosoph Michel de Certeau I980 in seinem Buch Linvention du quotidien (dt.: Kunst des Handelns) voneinander unterschieden hat. De Certeau definiert dabei einmal eine Raumlogik, die auf eine "maîtrise des lieux par la vue « hinarbeitet: »La partition de l'espace permet une pratique panoptique à partir d'un lieu d'où le regard transforme les forces étrangères en objets (also ein abstrahierender Vorgang der Versachlichung, H. K.) qu'on peut observer et mesurer, contrôler donc et inclure dans sa vision $"{ }^{29}$ Dies kann dann z. B. in »cartes ${ }^{30}$ resultieren: "Voir (loin), ce sera également prévoir, devancer le temps par la lecture d'un espace. ${ }^{31}$

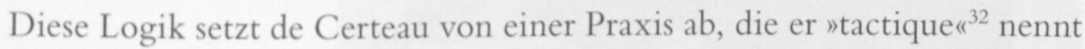
und die ohne einen solchen sicheren und kalkulierenden Sicherheitsabstand verfährt und sich stattdessen durch den Raum bewegt, indem sie diesen erst begreift und versteht, indem sie darin einen Schritt nach dem anderen setzt und sich (wie ein Blinder also) vorantastet, ohne jene selbstbewusste Kontrolle und "maîtrise des lieux par la vue" zu haben: "Elle n'a pas le moyen de se tenir en elle-même, à distance, dans une position de retrait, de prévision et de rassemblement de soi. (...) Elle n'a donc pas la possibilité de se donner un projet global ni de totaliser l'adversaire dans un espace distinct, visible et objectivable. Elle fait du coup par coup. Elle profite des soccasions et en dépend. « ${ }^{33}$

Das von de Certeau als "parcours« bezeichnete und von der zuvor beschriebenen Praxis der "cartes" unterschiedene Verfahren ${ }^{34}$ "lui permet sans doute la mobilité, (...) pour saisir au vol les possibilités qu' offre un instant. (...) Elle y crée des surprises. ${ }^{35}$

Also: Auf der einen Seite die Distanznahme und dadurch gewonnene Kontrolle durch stillstellende Versachlichung; auf der anderen Seite das SichEinlassen auf die konkreten Phänomene.

Und schließlich, so kann man feststellen, schlägt das Pendel von der Versachlichung in $M$ auch zurück in den extremen Gegenpol, in die Subjekti- 
vierung von Objekten: Ganz im Sinne von Strauss' Hitchcock's Objects as Subjects gibt es in Langs $\mathrm{M}$ durchaus auch jene Momente, in denen Objekte - noch über die beschriebene Dynamisierung der Schaufensterauslagen hinaus - fast zu Individuen werden, so etwa in der Tribunalszene, wenn der Ballon, der Beckert an seinen zu Beginn des Films an der kleinen Elsie Beckmann begangenen Mord erinnern soll (er hațte ihn dem Mädchen gekauft, um sich bei ihr einzuschmeicheln), etwas von einem Rachegeist bekommt, vor dem Beckert entsetzt zurückweicht. ${ }^{36}$

\section{VI. $\quad M-$ unbewegt}

Es ist nun interessant, sich abschließend auch anzuschauen, was passiert, wenn die in bewegten Bildern erzählte Handlung von Fritz Langs Film M in das mit unbewegten Bildern operierende Medium der Graphic Novel übertragen wird: 1990 und dann noch einmal 2008 in einer überarbeiteten Version adap-

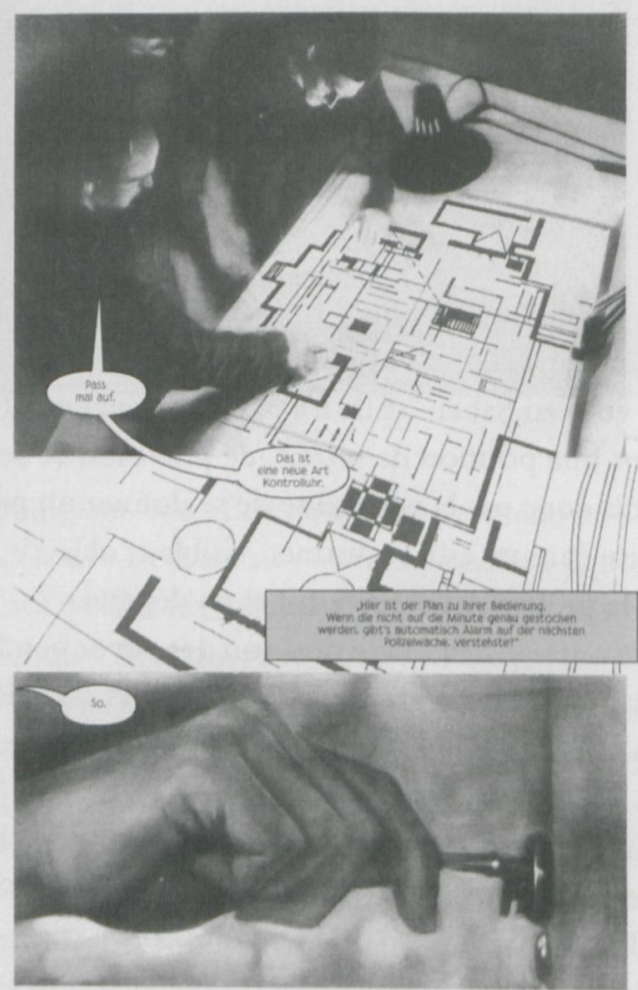

m)
Abb. $8 a-c$ :

Jon J Muth: M (2008), hier Ausschnitte aus der deutschen Lizenzausgabe der »Süddeutsche Zeitung Bibliothek«, München 2013, mit einer Montage aus Motiven von S. 51, 82, 127 (Bilder und Texte von Jon J Muth, ursprünglich bei Abrams ComicArts verlegt) 
Abb. 8 b

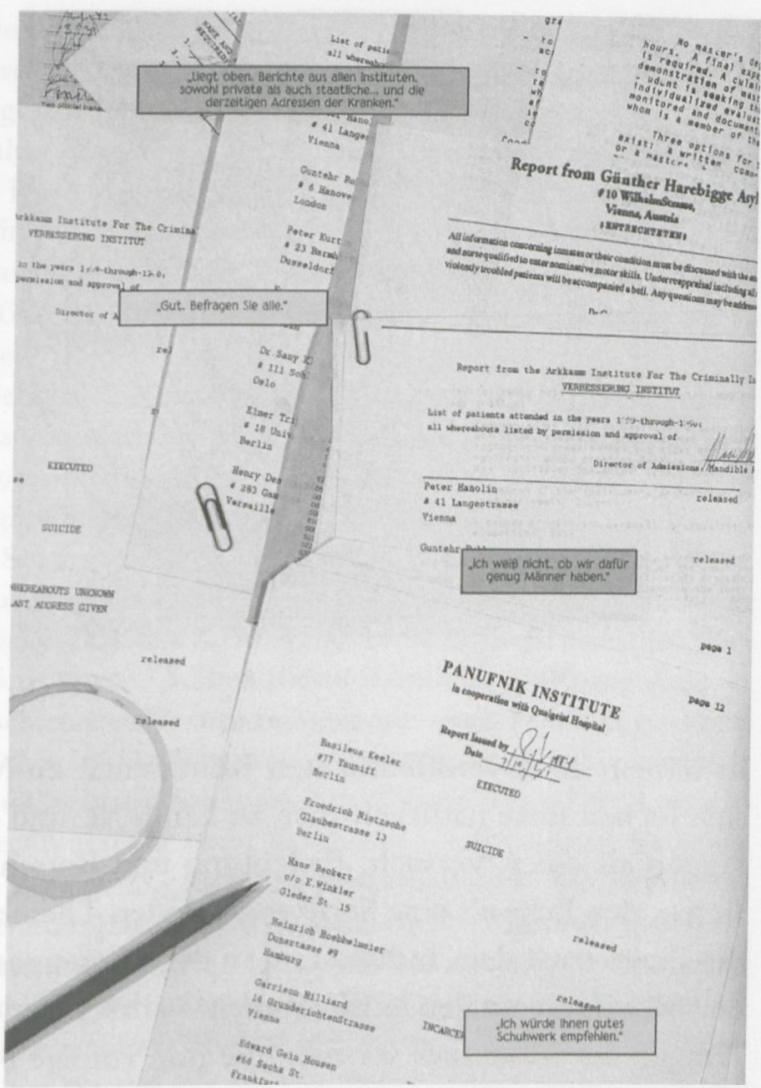

tierte der amerikanische Comic-Künstler und Kinderbuchillustrator Jon J Muth M als Graphic Novel. ${ }^{37}$ Er hat sich dabei in vielerlei Hinsicht von Langs Film emanzipiert, d.h. er hat diesen nicht einfach anhand statischer Bilder nachillustriert, sondern hat dessen Handlung sorgfältig und sehr überlegt modernisiert und adaptiert. Insofern versetzt er die Handlung nicht nur in eine eher zeitgenössische Stadt und verändert das Erscheinungsbild der Protagonisten, sondern er findet immer wieder auch passende Alternativen zu den Filmbildern Langs (vgl. z. B. die dramaturgisch ganz anders gehandhabte Szene mit dem Blinden und seinem Ballon am Schluss). Aber trotz all dieser Änderungen, Zusätze und Auslassungen gegenüber dem Film scheint auch Muth das Motiv der Karten und Pläne als so fundamental für die erzählte Geschichte verstanden zu haben, dass er sie immer wieder in seine Geschichte einbaut. Somit finden wir bei ihm nicht nur Stadtpläne oder Schaltpläne elektrischer Apparaturen, sondern auch das Motiv der zueinander in Beziehung gesetzten Listen und Akten $(\mathrm{Abb} .8 \mathrm{a}-\mathrm{c}) .^{38}$ 


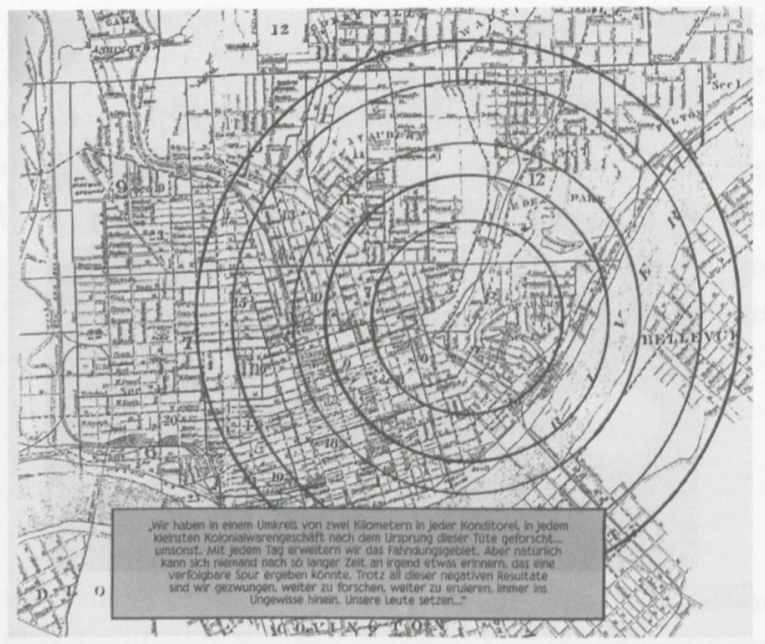

Abb. $8 c$

In seinem 2009 veröffentlichten Kommentar zu Muths Adaption ${ }^{39}$ äußert sich Jochen Ecke natürlich auch zu Langs M, und er versteht Muths Unterfangen als einen Versuch, Bedeutung und Botschaft des Films zu aktualisieren, den Ecke als eine Serie von "großen Themen der Moderne a auffasst: die Frage nach dem Individuum in der Massengesellschaft, die Frage nach der Moral, die quälende Einsamkeit - alles Themen, die Ecke zugleich als "Fragen der Moderne" versteht, die ihm zufolge bislang unbeantwortet geblieben sind. ${ }^{40}$

Vor diesem Hintergrund können die Pole der Dynamisierung des Unbelebten auf der einen und der Versachlichung und Stasis als Zeichen von dessen Kontrolle auf der anderen Seite in Fritz Langs $M$ auch als an uns gerichtete Fragen verstanden werden, wie wir heute mit Versachlichung (als Zeichen und Strategien der Kontrolle) sowie deren Konsequenzen umgehen wollen und wie bzw. ob wir uns dazu (im Sinne von de Certeaus "maîtrise des lieux par la vue») stellen oder (im Sinne von de Certeaus »tactique») bewegen.

1 Vgl. auch Einträge und Erwähnungen des Films jüngeren Datums in so verschiedenen Texten wie z. B. Michael Töteberg, "M - Eine Stadt sucht einen Mörder«, in: Metzler Filmlexikon, hg. von dems., Stuttgart/Weimar I995, S. 349-35I, hier S. 350 ("Streckenweise wird $M$ zur ,Film-Reportage «), oder Helmut G. Asper, "Im Märchenland. Fritz Langs und Thea von Harbous 'Spione«, in: Film-Dienst 7 (2005), S. 47 ( $[$ [...] obwohl ,Spione auf einem Zeitungsbericht basiert über die Entlarvung der 
russischen Handelsdelegation Arcos in London durch Scotland Yard als Spionagezentrum, so ist der Film andererseits auch meilenweit entfernt von der I93I in >M verwirklichten Film-Reportage «), oder einer Internetseite mit Ausschnitten aus einem Vortrag des Schweizer Kulturwissenschaftlers Thomas Barfuss von 2003 mit dem Titel "Spießerdämmerung. Die Modernisierung des Spießers im Film«, http://www. gegenentwurf-muenchen.de/thobarspiz.htm (letzter Zugriff am 7.I0.2016) (»Nach Langs Heroen- und Monumentalfilmen [Die Nibelungen I923/24 und Metropolis I926] ist $M$ - der erste Tonfilm des Regisseurs - eine 'Film-Reportage in der Tradition der >Neuen Sachlichkeit»). - 2 Hier referiert nach der Wiedergabe des Interviews in Noël Simsolo/Bernard Eisenschitz/Gérard Legrand, M le Maudit, Paris 1990, S. 24. - 3 Vgl. Thea von Harbou, "Warum gerade ein solcher Film ?!«, in: Berliner Volkszeitung, I2.5.I93I, hier zitiert nach dem Wiederabdruck in Christoph Bareither/Urs Büttner (Hg.), Fritz Langs M-Eine Stadt sucht einen Mörder. Texte und Kontexte, Würzburg 2010, S. 201-202. - 4 Vgl. dazu u.a. Martin Gehring, "Eine Mörderjagd in Bild und Ton. Fritz Langs $>M$ ` aus technischer Sicht», in: Fritz Langs M-Eine Stadt sucht einen Mörder. Texte und Kontexte, hg. von Christoph Bareither/Urs Büttner, Würzburg 2010, S. 33-42. - 5 Eben diesen Terminus griff Lang dann in einem zwei Tage später erschienenen Text prominent auf - vgl. Fritz Lang, »Mein Film M - ein Tatsachenbericht", in: Die Filmwoche 2I (I93I), S. 655-657, hier rezipiert nach dem Wiederabdruck in Christoph Bareither/Urs Büttner (Hg.), Fritz Lang MEine Stadt sucht einen Mörder, Würzburg 2010, S. I83-184. - 6 Siegfried Kracauer, "Unterwelt", in: Frankfurter Zeitung, I8.5.I93I, hier konsultiert nach dem Wiederabdruck in Christoph Bareither / Urs Büttner (Hg.), Fritz Lang M-Eine Stadt sucht einen Mörder, Würzburg 2010, S. 209-210. - 7 Vgl. Lotte Eisner, Fritz Lang, London 1976, S. II 4-II7, mit ihrer wiederholten Betonung des "documentary element in M" (ebd., S. II4ff.), dessen "cool, precise manner" (ebd., S. II6) jenen Realismus ermögliche "which Lang required». Als ein Beispiel des direkten Niederschlags dieser Sicht vgl. Douglas Pye / Iris Luppa, "M: Leading the Blind ", in: Movie 2 (20II), S. I-I3, hier S. 7: "The commissioner's narration places all the episodes he describes in what appears to be a factual context (the style draws on the contemporary Neue Sachlichkeit [New Objectivity] movement). As Lotte Eisner, among others, suggests, the whole section has a documentary feel to it (...): we are presented with apparently objective accounts (...). Eisner übersieht die starke filmische Stilisierung in M freilich nicht, jedoch ist sie bestrebt, diese auf gleich zwei Weisen zu relativieren: Zum einen setzt sie einzelne Szenen (z. B. Lotte Eisner, Fritz Lang [vgl. Anm. 7], S. II6: wthe shots of the police raids in the allotment gardens") davon ab, bei denen sthe camerawork itself is designed to produce the impression that newsreel material has been used ; zum anderen versucht sie, diese angeblich nüchtern gefilmten Szenen mit jenen in syntagmatische Beziehung zu setzen, in denen eine starke Stilisierung unleugbar ist, wobei das Credo: "The documentary and the ornamental are indivisible" (ebd.) als Leitspruch fungiert. Dahinter steht die taktische Argumentation, dass die visuell stilisierten Szenen niemals sonly playful and gratuitous (ebd.) seien, weshalb ihr zufolge selbst in jenen Aufnahmen "the impression that newsreel material has been used " vorherrscht, "where the pictorial composition is emphasized " (ebd.) - eine in meinen Augen sehr diskutable These, für die Eisner nicht nur den konkreten Beleg schuldig bleibt (die auf bestimmte Aussagen hin zugespitzten Kompositionen vertragen sich eigentlich wenig 
mit der Ästhetik von "newsreel material«), sondern die auch nicht konsequent begründet ist: Dass die Stilisierung nicht einfach nur ein formales, willkürliches Spiel ist, bedeutet im Umkehrschluss nicht zwingend, dass sie der "cool, precise manner von "newsreel material" zuzuschlagen sei. $\mathbf{- 8}$ Vgl. dazu auch Anton Kaes, $M$, London 200I, S. 29-35, sowie François Albera/Claire Angelini/Martin Barnier, "M/Le Maudit, ses doubles et son doublage", in: Décadrages 23-24 (2013), S. 80-1I3, online unter http://decadrages.revues.org/702 (letzter Zugriff am 7.10.20I6), hier S. 89-100. In einem bis 2010 unpublizierten Typoskript, das sich in der Fritz Lang Collection der Cinematic Arts Library an der University of Southern California, Los Angeles, befindet, schildert Lang, dass es der Fall »Kürten« war, der ihn überhaupt erst auf die Idee zu M brachte, dass er Zugang zu nthe communications and secret publications of the Berlin Police Department on Alexanderplatz " erhielt, "and so was able to document exactly the police procedure used to capture such a criminal", und dass er sogar noch während der Dreharbeiten immer wieder Inspirationen aus den seinerzeit noch laufenden Ermittlungen im Fall „Kürten« bezog. Vgl. Fritz Lang, "Some random notes about `M « which may be of interest to students of film", in: Fritz Lang $M$ - Eine Stadt sucht einen Mörder, hg. von Christoph Bareither/Urs Büttner, S. I86-I87. 9 Vgl. hierzu z.B. Bénédicte Savoy, Vom Faustkeil zur Handgranate. Filmpropaganda für die Berliner Museen 1934-1939, Köln/Weimar/Wien 20r4, die u.a. auf S. I24 das Drehbuch zu dem Dokumentarfilm HeIlbehandlung von Kunstwerken von Hans Cürlis aus dem Jahr 1939 abbildet und auf S. I23-I24 bespricht. - 10 Bill Nichols, Introduction to Documentary, Indiana 200I, insb. S. I-5 und 36. - 11 John Grierson, "Flaherty's Poetic Moana«, in: New York Sun, 8.2.1926. - 12 John Grierson, "First Principles of Documentary" (erstmals in: Cinema Quarterly 1.2 [Winter 1932], S. 67-72), hier zitiert nach: Catherine Fowler (Hg.), The European Cinema Reader, London 2002, S. 39-44, hier S. 39. - 13 Vgl. Anton Kaes, M (s. Anm. 8), S. 46-49. 14 Marc Raymond Strauss, Hitchcock's Objects as Subjects. The Significance of Things on the Screen, Jefferson (North Carolina) 20r6. Eben diese Verwendung, bei der den konkreten Dingen eine bestimmte Funktion übertragen wird, steht auch hinter dem in diesem Text gepflegten Verständnis von "Ding « und "Sache«: Während das "Ding" meistens auf etwas Konkretes zielt, tendiert der Gebrauch von "Sache " dazu, eher Abstraktes zu bezeichnen, d.h. in dem Moment, in dem z. B. von Individuen abstrahiert wird, werden diese "versachlicht $«$. Wie sich sowohl an Strauss' Analyse als auch an den Szenen in Langs M zeigt, in denen Dinge dynamisiert werden (siehe hierzu auch Anm. 26), wird Dingen demgegenüber zuweilen eher ein sie fast individualisierendes Eigenleben zugeschrieben, mit dessen Hilfe sie (vgl. die entsprechenden Szenen bei Hitchcock oder in Langs $M$ ) bestimmte Gefühlslagen artikulieren und so geradezu personifizieren können. - $15 \mathrm{Vgl}$. dazu ebd., S. 19-26. - 16 Ebd., S. 76-78. - 17 Denis Wood, The Power of Maps, New York/London 1992; Denis Wood, Rethinking the Power of Maps, New York/London 20Io. - 18 John Brian Harley, "Deconstructing the Map«, in: Cartographica 26.2 (I989), S. I-20. - 19 John Brian Harley, "Deconstructing the Map", in: Writing Worlds: Discourse, Text and Metaphor in the Representation of Landscape, hg. von Trevor J. Barnes/James S. Duncan (Hg.), London 1992, S. 23I-247, hier S. 244. - 20 Fritz Lang, M, DVD-Ausgabe von EUREKA-Video, 2003, 0:15:19-0:19:52. - 21 Siehe hier Anm. 8. - 22 Fritz Lang, $M$ (s. Anm. 20), 0:33:35-0:33:40. - 23 Ebd., 0:39:58-0:40:01. - 24 Vgl. dazu insbe- 
sondere den Abschnitt "Sound as a dramatic factor" bei Lotte Eisner, Fritz Lang (s. Anm. 7), S. I17-I27. - 25 Fritz Lang, M(s. Anm. 20), 0:44:20-0:44:25. - 26 Vgl. Anton Kaes, $M$ (s. Anm. 8), S. 59-6I, wo die Schaufensterdekorationen als novertly symbolic bezeichnet werden. Kaes interpretiert die Auslage mit dem auf und ab schnellenden und so auf das darunter vorbeilaufende Kind deutenden Pfeil schon aufgrund des "phallic arrow" als voller "strong sexual associations«: "In $M$ the manically moving objects correspond to Beckert's agitated state. His feral desire and lust radiate outward, animating all objects around him ". Um diese und die daraus erwachsenden Konsequenzen demgegenüber dingfest machen und in ihre Schranken weisen zu können, bedarf die Polizei des Mittels der Erstarrung und Fixierung des Lebendigen (vgl. auch hier Anm. I4). - 27 Fritz Lang, $M$ (s. Anm. 20), 0:50:44-0:51:48 u. I:00:25-I:0r:03. - 28 Ebd., 0:I6:35-0:17:03. - 29 Michel de Certeau, L'invention du quotidien. 1. Arts de faire, Paris I990, S. 60. In der deutschen Übersetzung (Berlin I988, S. 88): "Die Gliederung des Raumes ermöglicht eine panoptische Praktik ausgehend von einem Ort, von dem aus der Blick die fremden Kräfte in Objekte verwandelt, die man beobachten, vermessen, kontrollieren und somit seiner eigenen Sichtweise seinverleiben kann. - $\mathbf{3 0}$ Vgl. so auch die Überschrift eines Abschnitts bei de Certeau. Ebd., S. 175: "Parcours et cartes." -31 Ebd., S. 6o. In der deutschen Übersetzung (Berlin I988, S. 88): "Sehen (in die Ferne sehen), bedeutet auch voraussehen, also durch die Lektüre der Zeit vorauseilen." - 32 Ebd. - 33 Ebd., S. 60-6I. In der deutschen Übersetzung (Berlin I988, S. 89): "Sie ist nicht in der Lage, sich bei sich selbst aufzuhalten, also auf Distanz, in einer Rückzugsposition, wo sie Vorausschau üben und sich sammeln kann (...). Sie hat also nicht die Möglichkeit, sich einen Gesamtüberblick zu verschaffen und den Gegner in einem abgetrennten, überschaubaren und objektivierbaren Raum zu erfassen. Sie macht einen Schritt nach dem anderen. Sie profitiert von , Gelegenheiten und ist von ihnen abhängig. « - $\mathbf{3 4} \mathrm{Vgl}$. hier Anm. 30. - 35 Michel de Certeau, Linvention (s. Anm. 29), S. 6I. In der deutschen Übersetzung (Berlin I988, S. 88): "(...) ermöglicht zweifellos die Mobilität (...), um im Fluge die Möglichkeiten zu ergreifen, die der Augenblick bietet. (...) Sie (...) sorgt für Überraschungen." - 36 Fritz Lang, $M$ (s. Anm. 20), 1:33:00-1:33:I7. 37 Die Graphic Novel erschien zunächst in vier Heften als: Jon J Muth, $M$, Forestville (California) I990, und dann noch einmal 2008 überarbeitet als einbändige Ausgabe: Jon J Muth, M, New York 2008. Muths Graphic Novel wurde sowohl 1992 (Feest Comics, Stuttgart) als auch 2009 (Cross Cult, Ludwigsburg) ins Deutsche übertragen. Für diesen Beitrag wurde mit der deutschen Lizenzausgabe der »Süddeutsche Zeitung Bibliothek", München 2013 gearbeitet. - 38 Jon J Muth, $M$ - Eine Stadt sucht einen Mörder, München 20I3, S. 5I, 82 und I27. - 39 Jochen Ecke, "Auf der Suche nach dem Neuen im Alten - Fritz Langs und Jon J Muths >M ( (193I/I990)«, in: Jon J Muth, M-Eine Stadt sucht einen Mörder, München 2013, S. 203-208. 40 Ebd., S. 208. 